\title{
Disc prolapse in pregnancy
}

\section{Sangeeta Arya, Nidhi Tripathi, Amrita Singh*}

Department of Obstetrics and Gynaecology, GSVM Medical College, Kanpur, Uttar Pradesh, India

Received: 23 August 2016

Revised: 30 September 2016

Accepted: 01 October 2016

\author{
*Correspondence: \\ Dr. Singh Amrita, \\ E-mail: amritanitinshankar@gmail.com
}

Copyright: (c) the author(s), publisher and licensee Medip Academy. This is an open-access article distributed under the terms of the Creative Commons Attribution Non-Commercial License, which permits unrestricted non-commercial use, distribution, and reproduction in any medium, provided the original work is properly cited.

\begin{abstract}
Surgery is required only in about 1 in 10 cases of slipped disc. It may be considered if there is evidence of severe nerve compression, symptoms not improved using other treatments, having difficulty in standing or walking, have severe symptoms such as progressive muscle, weakness or altered bladder function. A microdiscectomy is typically performed for a herniated lumbar disc and is actually more effective for treating leg pain (also known as radiculopathy) than lower back pain. Impingement on the nerve root (compression) can cause substantial leg pain. While it may take weeks or months for the nerve root to fully heal and any numbness or weakness to get better, patients normally feel relief from leg pain almost immediately after a microdiscectomy spine surgery. Backache during pregnancy must not be overlooked as only minor ailment due to mechanical stress. Patient should be investigated on lines of spinal pathology.
\end{abstract}

Keywords: Disc prolapse, Pregnancy, Backache, MRI spine

\section{INTRODUCTION}

A prolapsed (herniated) disc occurs when the outer fibres of the intervertebral disc (its annulus) are injured and soft material known as nucleus pulposus, ruptures out of its enclosed space. The prolapsed or ruptured disc material can enter the spinal canal, compressing the spinal cord, but more frequently spinal nerve, most common in young and middle aged adults. Pregnancy in some way predisposes to the development of a lumbar disc protrusion. ${ }^{1}$

Most commonly occur in the lumbar spine and cervical spine, less commonly, they occur in thoracic spine.

\section{CASE REPORT}

A 26 old female presented in our antenatal clinic in first trimester with complains of back pain, which was insidious in onset dating back to 3-4 months prior to conception. Intermittent in nature, radiating to left side of gluteal region and thigh, responding to analgesics. Along with analgesics, patient was advised physiotherapy and other chiropractic measures from our side. However pain persisted and increased in intensity with advancing gestation. At 37 completed weeks, patient was planned for elective cesarean section because of this backache in order to prevent her from subjecting to cumbersome lithotomy position during labour. She underwent uneventful cesarean delivery with a healthy male child.

On her $2^{\text {nd }}$ post-operative day when patient was asked to ambulate, she again had backache which was now more severe in intensity, radiating to whole of left side of lower body since her $5^{\text {th }}$ post-operative day, patient developed weakness in her left side of lower body. Her orthopedic reference was done and patient was advised X-Ray Pelvis and MRI of spine. X-Ray was normal however MRI was suggestive of disc prolapse at L5-S1 level patient was discharged from our side on $8^{\text {th }}$ post-operative day for further management of her disc prolapse.

She was operated for the same-procedure was micro diskectomy under general anaesthesia and was discharged on next day of procedure. Thereafter patient had drastic pain relief and can move better. 


\section{DISCUSSION}

Postural stress during pregnancy and mechanical stress during labour may lead to injury of lumbar-inter vertebral joint, further diminution of lumbar muscle tone in the puerperium may increase risks of such Injury from minor stress. A secretion of corpus luteum, relaxin, has been thought to be responsible for the increased mobility of sacro iliac joint and pubic symphyseal joints during pregnancy. It seems that this secretion might also induce changes in the ligamentous coverings of other joints, including lumbar inter vertebral ones and that such changes might render these joints more vulnerable to stress. $^{2}$

Moreover, since such a protrusion may occur early in the puerperium and produce severe limb paresis. Also differentiation of paresis due to plexus injury from those due to disc protrusions should present little difficulty clearly when paralysis and sensory loss develop with little or no associated pain and without spinal signs or evidence of tension in the roots of the sciatic nerve, the diagnosis is likely to be one of a plexus injury during labour when, on the contrary the neurological defect is associated with much pain and with well-marked spinal and tension signs the diagnosis of a lumbar disc protrusion is the likely one. However pain is recorded as having severe in some cases considered being examples of plexus injury and it is common experience that when a lumbar disc protrusion causes severe Nerve injury and paralysis, pain due to it may greatly diminish.

Surgery is required only in about 1 in 10 cases of slipped disc. It may be considered, if:

- There is evidence of severe nerve compression.

- Symptoms not improved using other treatments

- Having difficulty in standing or walking.

- Have severe symptoms. Such as progressive muscle.

- Weakness or altered bladder function.

Microdiscectomy is a very safe procedure and can be performed even during antenatal period. In a microdiscectomy or microdecompression spine surgery, a small portion of the bone over the nerve root and/or disc material from under the nerve root is removed to relieve neural impingement and provide more room for the nerve to heal. ${ }^{3}$

A microdiscectomy is typically performed for a herniated lumbar disc and is actually more effective for treating leg pain (also known as radiculopathy) than lower back pain.

Impingement on the nerve root (compression) can cause substantial leg pain. While it may take weeks or months for the nerve root to fully heal and any numbness or weakness to get better, patients normally feel relief from leg pain almost immediately after a microdiscectomy spine surgery. ${ }^{4,5}$

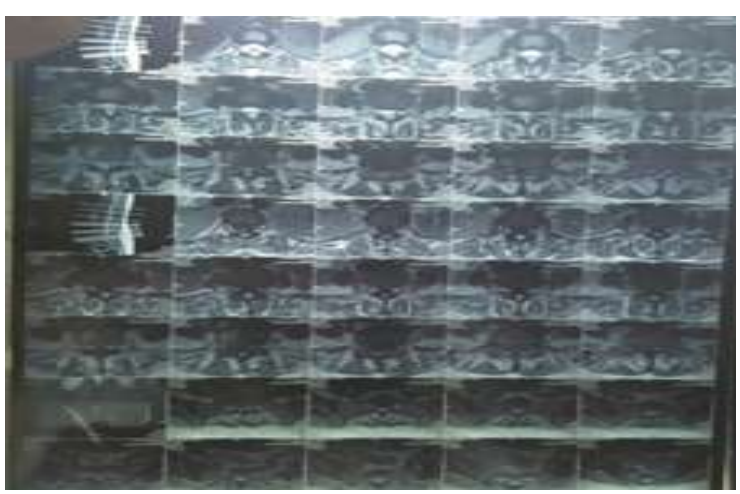

Figure 1: Sections of MRI whole spine.

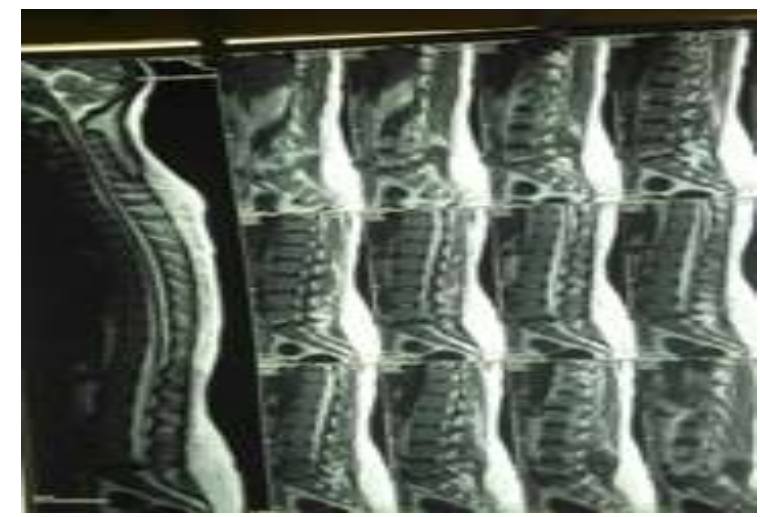

Figure 2: MRI cervico-thoracic and lumbo-dorsal spine.

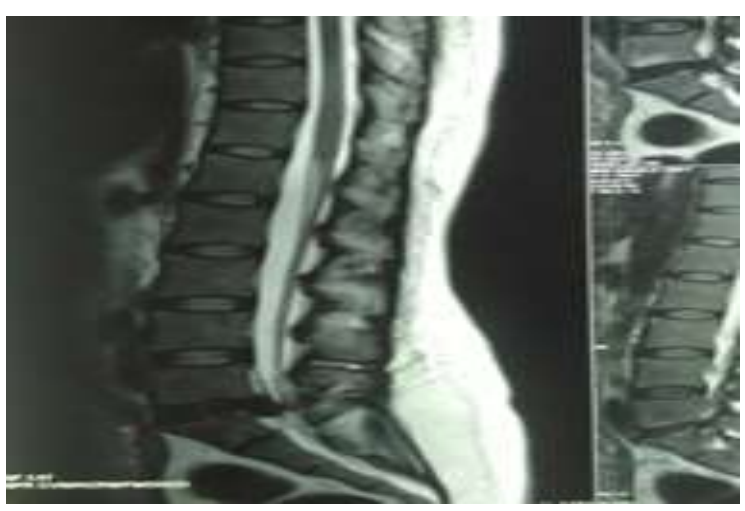

Figure 3: Disc prolapse at the level L5-S1.

\section{CONCLUSION}

In conclusion backache during pregnancy must not be overlooked as only minor ailment due to mechanical stress. Patient should be investigated on lines of spinal pathology.

Funding: No funding sources

Conflict of interest: None declared

Ethical approval: Not required 


\section{REFERENCES}

1. Han IH. Pregnancy and spinal problems. Curr Opin Obstet Gynecol. 2010;22(6):477-81.

2. Mohapatra RN, Patra RK. Journal, Indian Academy of Clinical Medicine. 2008;9(2).

3. Jacobs WCH, Maurits van T, Peul WC. Surgery versus conservative management of sciatica due to a lumbar herniated disc: a systematic review, Eur. Spine Journal. 2011:20(4):513-22.
4. Price C, Arden N, Coglan L, Rogers P. Costeffectiveness and safety of epidural steroids in the management of sciatica. Health Technol Assess. 2005;9:1-58.

5. Valat JP, Genevay S, Marty M, Rozenberg S, Sciatica KB. Best Pract Res Clin Rheumatol. 2010;24:241-52.

Cite this article as: Arya S, Tripathi N, Singh A. Disc prolapse in pregnancy. Int J Reprod Contracept Obstet Gynecol 2016;5:4067-9. 